\title{
Generalising Wigner's theorem
}

\author{
Gniewomir Sarbicki and Dariusz Chruściński \\ Institute of Physics, Faculty of Physics, Astronomy and Informatics \\ Nicolaus Copernicus University, \\ Grudziądzka 5/7, 87-100 Toruń, Poland \\ Marek Mozrzymas \\ Institute for Theoretical Physics, University of Wrocław \\ 50-204 Wrocław, Poland
}

August 8, 2021

\begin{abstract}
We analyse linear maps of operator algebras $\mathcal{B}_{H}(\mathcal{H})$ mapping the set of rank- $k$ projectors onto the set of rank- $l$ projectors surjectively. A complete characterisation of such maps for prime $n=\operatorname{dim} \mathcal{H}$ is provided. A particular case corresponding to $k=l=1$ is well known as the Wigner's theorem. Hence our result may be considered as a generalization of this celebrated Wigner's result.
\end{abstract}

\section{Introduction}

The celebrated Wigner's theorem [1] in its original formulation says that any map $\Phi$ between rank-1 projectors in a Hilbert space preserving the Hilbert-Schmidt product, i.e. $\left(\Phi\left(P_{1}\right), \Phi\left(P_{2}\right)\right)_{\mathrm{HS}}=\left(P_{1}, P_{2}\right)_{\mathrm{HS}}$, is of the form:

$$
\Phi(X)=U X U^{\dagger} \text { or } \Phi(X)=U X^{\mathrm{t}} U^{\dagger},
$$

where $X^{\mathrm{t}}$ denotes a transposition with respect to a fixed orthonormal basis in $\mathcal{H}$ and $U$ is a unitary operator (see also [2] and recent analysis in [3]). It is clear that any such map induces unitary or antiunitary operation in the original Hilbert space. The Wigner's theorem is sometimes reformulated as follows [6]: one restricts to linear maps $\Phi: \mathcal{B}(\mathcal{H}) \rightarrow \mathcal{B}(\mathcal{H})$ which are not necessarily Hilbert-Schmidt isometries. Now, any such map that maps bijectively rank-1 projectors to rank-1 projectors is of the form (1). Clearly, a map mapping rank-1 projectors to rank-1 projectors is by construction a positive map [4, 5] and hence the Wigner's theorem states that a positive trace-preserving map $\Phi$ has a positive inverse if and only it has a form (11).

In this paper we consider linear maps mapping surjectively rank- $k$ projectors to rank- $l$ projectors. We show for which $k, l$ such maps exist and that for prime dimensions of the Hilbert space they has exactly the form (11). We also provide an example of a map which is not of the form (11). Interestingly, linear maps acting surjectively between sets of projectors of fixed rank maps have recently attracted attention in the problem of entanglement detection [7].

Let us consider a real space of self-adjoint operators $\mathcal{B}_{H}(\mathcal{H})$. We denote the set of rank- $k$ projectors supported on a subspace $V \subset \mathcal{H}$ by $\mathcal{P}_{k}(V) \subset \mathcal{B}_{H}$. It is a smooth manifold of dimension $k(n-k)$. Let $\Phi$ be a linear endomorphism of $\mathcal{B}(\mathcal{H})$ and let $\Phi\left(\mathcal{P}_{k}(\mathcal{H})\right)=\mathcal{P}_{l}(\mathcal{H})$. From the fact, that $\mathcal{P}_{l}(\mathcal{H})$ spans the whole $\mathcal{B}(\mathcal{H})$, one gets that $\Phi$ is a surjective endomorphism, hence a bijection, hence also a bijection between sets $\mathcal{P}_{k}(\mathcal{H})$ and $\mathcal{P}_{l}(\mathcal{H})$. This implies, that $l=k$ or $l=n-k$. There is a natural linear isomorphism between $\mathcal{P}_{k}(\mathcal{H})$ and $\mathcal{P}_{n-k}(\mathcal{H})$ :

$$
R_{k}(X)=\frac{1}{k} \mathbb{I} \operatorname{Tr} X-X
$$


Hence any bijection $\Phi: \mathcal{P}_{k}(\mathcal{H}) \rightarrow \mathcal{P}_{n-k}(\mathcal{H})$ can be represented as a composition of $R_{k}$ and bijective endomorphism of $\mathcal{P}_{k}(\mathcal{H})$. Therefore, it suffices to characterise all bijective endomorphisms of $\mathcal{P}_{k}(\mathcal{H})$.

Note, that this problem belongs to so-called linear preserver problem which deal with characterization of linear operators that leave certain properties or certain subsets in its domain invariant. This program was started already by Frobenius [9]. A well known example of linear preservers are rank preserver, nilpotency preserver and spectrum preserver: $\Phi$ is rank preserver iff $\Phi(X)=M X N$ or $\Phi(X)=M X^{\mathrm{t}} N$, where $M, N$ are invertible elements from $M_{n}(\mathbb{C})$. $\Phi$ provides a preserver of nilpotency iff $\Phi(X)=M X N$ or $\Phi(X)=M X^{\mathrm{t}} N$, where $M, N \in M_{n}(\mathbb{C})$ such that $M N=c \mathbb{I}_{n}$ and $c \in \mathbb{C}$. Finally, $\Phi$ is a spectrum preserver iff $\Phi(X)=M X M^{-1}$ or $\Phi(X)=M X^{\mathrm{t}} M^{-1}$.

Clearly, $\Phi$ defined in (1) is an example of rank preserver and nilpotency preserver, but in our problem the restriction is weaker - we demand preserving the nilpotency only for a one given value of the rank (the set of nilpotent hermitian operators splits into connectivity components grouping the projectors of the same rank). With a weaker assumption one can expect that the resulting set of linear operation can be in general greater. Indeed, for a special choice of $n$ and $k$ we provide an example of an invertible map preserving rank- $k$ projector not being of the form (11).

Recently Marciniak [11 considered a related problem and shown that every positive map $\Phi$ such that $\operatorname{rank} \Phi(P) \leq 1$ for any rank-1 projector $P$ is the rank-1 preserver and has a form (1).

\section{Main result}

This section provides the main result of the paper. Let us start with the following

Proposition 1. Any $\Phi: \mathcal{B}_{H}(\mathcal{H}) \rightarrow \mathcal{B}_{H}(\mathcal{H})$ mapping bijectively $\mathcal{P}_{k}(\mathcal{H})$ onto itself preserves the orthogonality.

Proof. If $2 k>n$, then there are no non-zero mutually orthogonal elements in $\mathcal{P}_{k}(\mathcal{H})$ and the proposiion is true in a trivial way. We will consider the case when $2 k \leq n$. Let $P_{V}$ be the projector onto a $2 k$-dimensional subspace $V \subset \mathcal{H} . P_{V}$ can be decomposed in various ways into the sum of two rank- $k$ orthogonal projectors $P_{1}$ and $P_{2}$. They are mapped via $\Phi$ onto two rank- $k$ projectors $Q_{1}=\Phi\left(P_{1}\right)$ and $Q_{2}=\Phi\left(P_{2}\right)$. From the positivity of $Q_{1}$ and $Q_{2}$ one has $\operatorname{Im} Q_{i}=\operatorname{Im} \Phi\left(P_{i}\right) \subset \operatorname{Im} \Phi\left(P_{V}\right)$. One can repeat it for any choose of $P_{1}$ and $P_{2}$, hence $\forall P \in \mathcal{P}_{k}(V) \operatorname{Im} \Phi(P) \subset \operatorname{Im} \Phi\left(P_{V}\right)$ and thus $\Phi\left(\mathcal{P}_{k}(V)\right) \subset \mathcal{B}_{H}\left(\operatorname{Im} \Phi\left(P_{V}\right)\right)$. Because $\mathcal{P}_{k}(V)$ spans the whole $\mathcal{B}_{H}(V)$, we have that $\Phi\left(\mathcal{B}_{H}(V)\right) \subset \mathcal{B}_{H}\left(\operatorname{Im} \Phi\left(P_{V}\right)\right)$.

While $\Phi$ is bijective on $\mathcal{P}_{k}(\mathcal{H})$, it is bijective on $\mathcal{B}_{H}(\mathcal{H})$. Hence $\operatorname{dim} \mathcal{B}_{H}(V) \leq \operatorname{dim} \mathcal{B}_{H}\left(\operatorname{Im} \Phi\left(P_{V}\right)\right)$ and hence $\operatorname{dim} V \leq \operatorname{dim} \operatorname{Im} \Phi\left(P_{V}\right)$. While $\Phi\left(P_{V}\right)$ is a sum of two rank- $k$ projectors, $\operatorname{dim} \operatorname{Im} \Phi\left(P_{V}\right) \leq 2 k=\operatorname{dim} V$ and hence $\operatorname{dim} \operatorname{Im} \Phi\left(P_{V}\right)=\operatorname{dim} V$. $\Phi$ establishes a bijection between $\mathcal{B}_{H}(V)$ and $\mathcal{B}_{H}\left(\operatorname{Im} \Phi\left(P_{V}\right)\right)$ and hence between $\mathcal{P}_{k}(V)$ and $\mathcal{P}_{k}\left(\operatorname{Im} \Phi\left(P_{V}\right)\right)$. Any rank- $k$ projector $Q \in \mathcal{P}_{k}\left(\operatorname{Im} \Phi\left(P_{V}\right)\right)$ can be realised as $\Phi(P)$ for some $P \in \mathcal{P}_{k}(V)$.

Now we choose the basis of $\operatorname{Im} \Phi\left(P_{V}\right)$ to make $\Phi\left(P_{V}\right)$ diagonal. Take a rank- $k$ dimensional projector $Q \in \mathcal{P}_{k}\left(\operatorname{Im} \Phi\left(P_{V}\right)\right)$, diagonal in this base (commuting with $\left.\Phi\left(P_{V}\right)\right)$. The operator $\Phi\left(P_{V}\right)-Q=\Phi\left(P_{V}\right)-\Phi(P)=$ $\Phi\left(P_{V}-P\right)$ is a rank $k$-projector, diagonal in the chosen basis. One can easily find, that this implies, that only possible values on the diagonal of $\Phi\left(P_{V}\right)$ are $1 \mathrm{~s}$ and $2 \mathrm{~s}$. But the rank of $\Phi\left(P_{V}\right)$ and its trace are equal $2 k$, so $\Phi\left(P_{V}\right)$ is the projector onto $\operatorname{Im} \Phi\left(P_{V}\right)$.

For any two orthogonal projectors $P_{1}$ and $P_{2}$ the operator $\Phi\left(P_{1}+P_{2}\right)=\Phi\left(P_{1}\right)+\Phi\left(P_{2}\right)$ is a rank-2k dimensional projector, hence the projectors $\Phi\left(P_{1}\right)$ and $\Phi\left(P_{2}\right)$ are orthogonal

One has immediately the following:

Corollary 1. Any $\Phi: \mathcal{B}_{H}(\mathcal{H}) \rightarrow \mathcal{B}_{H}(\mathcal{H})$ mapping bijectively $\mathcal{P}_{k}(\mathcal{H})$ onto itself maps bijectively $\mathcal{P}_{\text {qk }}(\mathcal{H})$ onto itself for $q \in \mathbb{N}$.

Remark 1. Note, that Wigner's theorem immediately follows from preserving the orthogonality relation and the properties of a spectrum preserver. Indeed, preserving the orthogonality relation of rank-1 projectors 
implies that a Schatten decomposition $\sum_{i} \lambda_{i} P_{i}$ of a hermitian operator is mapped to another Schatten decomposition with the same spectrum. Such a map is therefore a spectrum preserver and hence has a form $\Phi(X)=M X M^{-1}$ or $\Phi(X)=M X^{\mathrm{t}} M^{-1}$. Finally, preservation of orthogonality implies that $M$ is unitary.

Proposition 2. Assume, that $k=n \bmod$ l. If any linear map $\Psi: \mathcal{B}_{H}(\mathcal{H}) \rightarrow \mathcal{B}_{H}(\mathcal{H})$ transforming $\mathcal{P}_{k}(\mathcal{H})$ onto itself bijectively is of the form (1), then also each map $\Phi: \mathcal{B}_{H}(\mathcal{H}) \rightarrow \mathcal{B}_{H}(\mathcal{H})$ transforming $\mathcal{P}_{l}(\mathcal{H})$ onto itself bijectively is of the form (1).

Proof. Let $\Phi: \mathcal{B}_{H}(\mathcal{H}) \rightarrow \mathcal{B}_{H}(\mathcal{H})$ maps bijectively $\mathcal{P}_{l}(\mathcal{H})$ onto itself. Let $n=k+q \cdot l$. Then due to Corollary 1. for any $P \in \mathcal{P}_{q l}(\mathcal{H})$ one has $\Phi(P) \in \mathcal{P}_{q l}(\mathcal{H})$. Now, any projector from $\mathcal{P}_{q l}(\mathcal{H})$ may be written as $I-P_{k}$ with $P_{k} \in \mathcal{P}_{k}(\mathcal{H})$ and hence

$$
\Phi\left(I-P_{k}\right)=\Phi(I)-\Phi\left(P_{k}\right)=: I-Q_{k},
$$

for some $Q_{k} \in \mathcal{P}_{k}(\mathcal{H})$. One has therefore

$$
Q_{k}=\Phi\left(P_{k}\right)-\Phi(I)+I
$$

The above relation defines a map $P_{k} \rightarrow Q_{k}$ transforming $\mathcal{P}_{k}(\mathcal{H})$ onto itself bijectively, so due to our assumption it can be written as

$$
Q_{k}=U \widetilde{P}_{k} U^{\dagger}
$$

where $\tilde{X}=X$ or $\tilde{X}=X^{\mathrm{t}}$. Finally

$$
\Phi\left(P_{k}\right)=\Phi(I)-I+U \widetilde{P}_{k} U^{\dagger}
$$

and by linearity it may be extended to the following linear map on $\mathcal{B}(\mathcal{H})$

$$
\Phi(X)=\frac{1}{k}[\Phi(I)-I] \operatorname{Tr} \mathrm{X}+U \widetilde{X} U^{\dagger} .
$$

In particular if $X=P_{l} \in \mathcal{P}_{l}(\mathcal{H})$ one has

$$
\Phi\left(P_{l}\right)=\frac{l}{k}[\Phi(I)-I]+Q_{l}=: D+Q_{l},
$$

where $Q_{l}=U \widetilde{P}_{l} U^{\dagger} \in \mathcal{P}_{l}(\mathcal{H})$. To complete the proof we need to show that $D=0$. Since $D+Q_{l}$ is a projector one has $\left(D+Q_{l}\right)^{2}=D+Q_{l}$ and hence

$$
D Q_{l}+Q_{l} D=D-D^{2}
$$

Taking into account that $\Phi$ is trace-preserving one has $\operatorname{Tr} D=0$ which implies $2 \operatorname{Tr}\left(D Q_{l}\right)=-\operatorname{Tr} D^{2}$ for all $Q_{l} \in \mathcal{P}_{l}(\mathcal{H})$. Now, if $Q_{l}$ is a projector on the $l$-dim. subspace spanned by eigenvectors of $D$ corresponding to $l$ largest eigenvalues of $D$, i.e $d_{1} \geq d_{2} \geq \ldots \geq d_{l}$, then $2 \operatorname{Tr}(Q D)=2 \sum_{i=1}^{l} d_{i}=-\operatorname{Tr} D^{2} \leq 0$ but since $D$ is traceless one has $\sum_{i=1}^{l} d_{i} \geq 0$ which proves that $D=0$.

The main result of the paper is provided by the following

Theorem 1. If $n$ is prime, then any $\Phi$ mapping surjectively rank- $k$ projectors into rank- $k$ projectors is of the form (1).

Proof. Let us define a sequence via formula $k_{i+1}=n \bmod k_{i}$ and $k_{0}=k$. This is strictly decreasing, finite sequence, and it terminates at 0 and let $0=n \bmod k_{*}$, that is, the sequence reads $\left\{k_{0}=k>k_{1}>k_{2}>\ldots>\right.$ $\left.k_{*}>0\right\}$. Due to the Proposition 2, if any $\Psi: \mathcal{B}_{H}(\mathcal{H}) \rightarrow \mathcal{B}_{H}(\mathcal{H})$ maps bijectively $\mathcal{P}_{k_{i+1}}(\mathcal{H})$ onto itself is of the form (11), then any $\Psi: \mathcal{B}_{H}(\mathcal{H}) \rightarrow \mathcal{B}_{H}(\mathcal{H})$ mapping bijectively $\mathcal{P}_{k_{i}}(\mathcal{H})$ onto itself is of the form (1) as well. Now, due to the Wigner's theorem, if $k_{*}=1$ then for any $k_{i}>k_{*}$ in the sequence, any $\Psi: \mathcal{B}_{H}(\mathcal{H}) \rightarrow \mathcal{B}_{H}(\mathcal{H})$ mapping bijectively $\mathcal{P}_{k_{i}}(\mathcal{H})$ onto itself is of the form (11), in particular $k_{0}=k$. Note, that $k_{*}$ is by construction a divisor of $n$ and hence if $n$ is prime, then $k_{*}=1$ which complete the proof. 
Observe, that $n=q_{i} k_{i}+k_{i+1}$ and if for some number $d$ one has $d \mid n$ and $d \mid k_{i}$ then $d \mid k_{i+1}$, so the common divisors of $n$ and $k_{0}=k$ are also the common divisors of all elements of the sequence. Thus this method does not give a conclusive answer if the starting point $k_{0}=k$ is not relatively prime to $n$. If $n$ and $k$ are relatively prime then a conclusive answer is not guaranteed. Indeed, if $k=3$ and $n=10$ one has the conclusive answer, but for $k=3$ and $n=8$ the metod does not give a conclusive answer. Having in mind, that $k_{*}$ is by construction a divisor of $n$, one has the following

Remark 2. To complete the characterisation of surjective maps from $\mathcal{P}_{k}(\mathcal{H})$ into $\mathcal{P}_{k}(\mathcal{H})$ it is enough to characterise these maps for $k \mid n$.

Remark 3. Let $n=2 k$. Consider

$$
\Phi(X)=\frac{\mathbb{I}_{2 k}}{k} \operatorname{Tr} X-X
$$

It is clear that $\Phi$ maps rank- $k$ projectors into rank- $k$ projectors but evidently it does not have the form (1). Indeed, if $P$ is rank-1 projector then $\Phi(P)$ has rank $n-1$ and hence it is not rank-1 projector. Note, that $\Phi^{-1}=\Phi$.

Such maps are the already defined (2) and because $\mathcal{P}_{k}(\mathcal{H})$ and $\mathcal{P}_{n-k}(\mathcal{H})$ are the same set, these maps are endomorphisms. This encourages us to claim that these are the only additional endomorphisms. Therefore we pose the following

Conjecture 1. Let $n=2 k$ and $\Phi: \mathcal{P}_{k}(\mathcal{H}) \rightarrow \mathcal{P}_{k}(\mathcal{H})$ surjectively. Then the map is of the form $\Phi$ or $\Phi \circ R_{k}$, where $\Phi$ is of the form (11) and $R_{k}$ is defined by (2).

This conjecture allows to perform perfect characterization of surjective maps $\mathcal{P}_{k}(\mathcal{H}) \rightarrow \mathcal{P}_{k}(\mathcal{H})$

Proposition 3. Let us assume that Conjecture 1 is true. If $k \mid n, n / k>2$ and $\Phi: \mathcal{P}_{k}(\mathcal{H}) \rightarrow \mathcal{P}_{k}(\mathcal{H})$ is surjective, then $\Phi$ has the form (1).

Proof. Let $\left\{e_{i}\right\}_{i=0}^{n-1}$ be the standard basis of $\mathcal{H}$ and let $P_{i}$ be a rank- $k$ projector defined by

$$
P_{i}=\sum_{j=0}^{k-1}\left|e_{i k+j}\right\rangle\left\langle e_{i k+j}\right|, \quad i=0,1, \ldots, \frac{n}{k}-1 .
$$

It is clear that $P_{i}$ and $P_{j}$ are mutually orthogonal for $i \neq j$. Now, due to the Proposition 1 , a set of projectors $\left\{P_{i}\right\}$ is mapped to the set of pairwise orthogonal rank- $k$ projectors. Now, since

$$
P_{0}+\ldots+P_{n / k}=\mathbb{I}=\Phi\left(P_{0}\right)+\ldots+\Phi\left(P_{n / k}\right)
$$

projectors $\left\{\Phi\left(P_{0}\right), \ldots, \Phi\left(P_{n / k}\right)\right\}$ are unitarly equivalent to $\left\{P_{0}, \ldots, P_{n / k}\right\}$, that is, $\Phi\left(P_{i}\right)=U P_{i} U^{\dagger}$ for some unitary $U$. Without loosing generality we may assume that $U=\mathbb{I}$, that is, $\Phi$ maps $P_{i}$ to $P_{i}$. Let $V_{i}=$ $\operatorname{span}\left\{e_{k \cdot i}, \ldots e_{k \cdot i+(k-1)}\right\}$ be the range of $P_{i}$ and let $\mathbb{I}_{i j}$ be the projector onto the subspace $V_{i} \oplus V_{j}$. Let

$$
\Phi_{i j}:=\mathbb{I}_{i j} \Phi \mathbb{I}_{i j}
$$

be a restriction of $\Phi$ to the subspace $V_{i} \oplus V_{j}$. By the Conjecture 1 it is of the form $\Phi_{i j}(X)=U_{i j} \widetilde{\Phi}_{i j}(X) U_{i j}^{\dagger}$, where $\widetilde{\Phi}_{i j}$ maps $X$ to $X, X^{t}, \frac{1}{k} \mathbb{I}_{i j} \operatorname{Tr} X-X$ or $\frac{1}{k} \mathbb{I}_{i j} \operatorname{Tr} X-X^{t}$. One has that for all $i, j \Phi_{i j}\left(P_{i}\right)=P_{i}$ and $\Phi_{i j}\left(P_{j}\right)=P_{j}$, hence for all $\alpha, \beta$

$$
\left[\begin{array}{cc}
\alpha P_{i} & \\
& \beta P_{j}
\end{array}\right]=U_{i j}\left[\begin{array}{ll}
\alpha P_{i} & \\
& \beta P_{j}
\end{array}\right] U_{i j}^{\dagger}=\left[\begin{array}{cc}
A & B \\
C & D
\end{array}\right]\left[\begin{array}{cc}
\alpha P_{i} & \\
& \beta P_{j}
\end{array}\right]\left[\begin{array}{cc}
A & B \\
C & D
\end{array}\right]^{\dagger}
$$

what implies that $U_{i j}$ is block-diagonal if $\widetilde{\Phi}_{i j}(X)=X$ or $X^{t}$ and $U_{i j}$ is block-antidiagonal if $\widetilde{\Phi}_{i j}(X)=$ $\frac{1}{k} \mathbb{I}_{i j} \operatorname{Tr} X-X$ or $\frac{1}{k} \mathbb{I}_{i j} \operatorname{Tr} X-X^{t}$. 
Moreover, if $\psi \in V_{i}$ then

$$
\Phi_{i j}(|\psi\rangle\langle\psi|)=\mathbb{I}_{i j} \Phi(|\psi\rangle\langle\psi|)
$$

does not depend on $j$, and similarly if $\phi \in V_{j}$ then

$$
\Phi_{i j}(|\phi\rangle\langle\phi|)=\mathbb{I}_{i j} \Phi(|\phi\rangle\langle\phi|)
$$

does not depend on $i$. It follows, that all $\Phi_{i j}$ does nor depend on $i, j$ and hence $\widetilde{\Phi}_{i j}(X)=X$ or $X^{t}$ (otherwise it could not give the same result for different $j$ s if $n / k>2$, as the reduction map has the information about the trace of the second block). Now we know that all $U_{i j}$ s are block-diagonal and again, because $\Phi_{i j}(|\Psi\rangle\langle\Psi|)$ has to give the same result for all $j$ s one gets that $U_{i j}=U_{i} \oplus U_{j}$.

Finally we get that

$$
\Phi(X)=U X U^{\dagger} \quad \text { or } \quad \Phi(X)=U X^{t} U^{\dagger}
$$

with $U=\bigoplus_{i=1}^{n / k} U_{i}$, which ends the proof.

\section{Conclusions}

Let us summarise the paper by the following remarks:

Remark 4. Let us observe that if we relax the condition that the map $\Phi$ is invertible then one may have maps from $\mathcal{P}_{k}\left(\mathbb{C}^{n}\right)$ to $\mathcal{P}_{l}\left(\mathbb{C}^{n}\right)$ with $l \neq n-k$. The well known example is provided by the Breuer-Hall map $\Phi_{\mathrm{BH}}: M_{2 n}(\mathbb{C}) \rightarrow M_{2 n}(\mathbb{C})$ defined as follows [12, 13, 14,

$$
\Phi_{\mathrm{BH}}(X)=\frac{1}{2(n-1)}\left(\mathbb{I}_{2 n} \operatorname{Tr} X-X-U X^{\mathrm{t}} U^{\dagger}\right)
$$

where $U$ is an arbitrary anti-symmetric $2 n \times 2 n$ matrix. $\Phi_{\mathrm{BH}}$ maps rank-1 projectors into projectors of rank $2(n-1)$. It is evident that $\Phi_{\mathrm{BH}}$ is not invertible.

Remark 5. Let us observe that maps (1) are characterized by the following property: $\Phi$ is positive and tracepreserving and $\Phi^{-1}=\Phi^{*}$, where the dual map $\Phi^{*}$ is defined by $(X, \Phi(Y))_{\mathrm{HS}}=\left(\Phi^{*}(X), Y\right)_{\mathrm{HS}}$. Interestingly, the map (10) is characterized by the following property: $\Phi$ is trace-preserving and $\Phi^{-1}=\Phi^{*}=\Phi$. It means that both (11) and (10) are isometries with respect Hilbert-Schmidt product and hence the corresponding eigenvalues satisfy $\left|\lambda_{i}\right|=1$.

On the other hand, any rank-1 projector $P$ can be decomposed as a combination o rank- $k$ projectors commuting with $P$ and hence any hermitian operator can be decomposed as a combination of $n$ commuting rank- $k$ projectors. This combination is mapped into another combination of rank- $k$ projectors (in general not commuting) with the same coefficients and hence there exists a common upper bound for the maximal eigenvalue of all invertible maps mapping $\mathcal{P}_{k}(\mathcal{H})$ to itself. While such maps form a group, their eigenvalues has to lie therefore on the unit circle and at least one of them is equal 1 . We stress that this property is not equivalent to being a Hilbert-Schmidt isometry. If one could prove that for any invertible map $\Phi$ mapping $\mathcal{P}_{k}(\mathcal{H})$ to itself its adjoint map $\Phi^{*}$ has the same property, then it would imply that this class is a subclass of Hilbert-Schmidt isometries.

Remark 6. The crucial difference between (10) and (10) is positivity. Let us recall [5] that a trace-preserving map is positive iff

$$
\|\Phi(X)\|_{1} \leq\|X\|_{1}
$$

for all self-adjoint elements $X$. It is clear that (10) is not positive and hence violates (12). Interestingly, for $X$ traceless one has

$$
\|\Phi(X)\|_{1}=\|-X\|_{1}=\|X\|_{1}
$$


In particular taking two density operators $\rho_{1}$ and $\rho_{2}$ one has

$$
\left\|\Phi\left(\rho_{1}-\rho_{2}\right)\right\|_{1}=\left\|\rho_{1}-\rho_{2}\right\|_{1},
$$

which means that (10) preserves the distinguishability between arbitrary quantum states. Clearly, (1) enjoys the same property.

Finally, it is hoped that presented result finds applications in quantum information theory (e.g. entanglement detection) and the analysis of symmetries of quantum systems (e.g the evolution of quantum systems).

\section{Acknowledgements}

We thank anonymous referees for valuable comments.

\section{References}

[1] E. P. Wigner, Group Theory, Academic Press, New York, 1959.

[2] G. Cassinelli, E. De Vito, P. J. Lahti, A. Levrero, The Theory of Symmetry Actions in Quantum Mechanics with an Application to the Galilei Group, Springer 2004.

[3] R. Simon, N. Mukunda, S. Chaturvedi, and V. Srinivasan, Two elementary proofs of the Wigner theorem on symmetry in quantum mechanics, Phys. Lett. A 372, 17 (2008): ibidem 378,13 (2014).

[4] V. Paulsen, Completely Bounded Maps and Operator Algebras, Cambridge University Press, 2003.

[5] R. Bhatia, Positive Definite Matrices, (Princeton University Press, 2006).

[6] F. Buscemi, G. M. D'Ariano, M. Keyl, P. Perinotti, R. Werner, Clean Positive Operator Valued Measures, J. Math. Phys. 46, 082109 (2005)

[7] M. Mozrzymas, A. Rutkowski, and M. Studziński, Using non-positive maps to characterize entanglement witnesses, J. Phys. A: Math. Theor. 48, 395302 (2015).

[8] Chi-Kwong Li and Nam-Kiu Tsing, Linear Preserver Problems: A Brief introduction and Some Special Techniques, Lin. Alg. Appl. 162-164, 217 (1992).

[9] G. Frobenius, Über die Darstellung der endlichen Gruppen durch lineare Substitutionen, Sitzungsber. Deutsch. Akad. Wiss. Berlin, 1897, pp. 994-1015.

[10] P. Botta, S. Pierce, and W. Watkins, Linear transformations that preserve the nilpotent matrices, Pacific J. Math. 104, 39 (1983).

[11] M. Marciniak On extremal positive maps acting between type I factors Noncommutative Harmonic Analysis with Applications to Probability II, Banach Center Publications, vol 69 Institute of Mathematics, Polish Academy of Science, Warsaw 2010

[12] H.-P. Breuer, Optimal Entanglement Criterion for Mixed Quantum States, Phys. Rev. Lett. 97, 0805001 (2006).

[13] H.-P. Breuer, Separability criteria and bounds for entanglement measures, J. Phys. A: Math. Gen. 39, 11847 (2006).

[14] W. Hall, A new criterion for indecomposability of positive maps, J. Phys. A: Math. Gen. 39, (2006) 14119. 